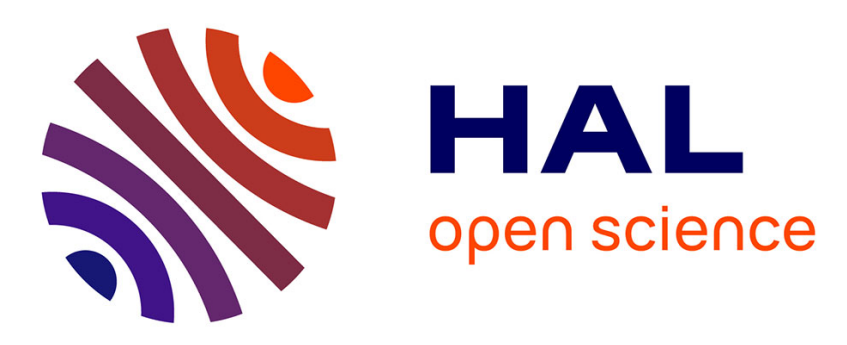

\title{
Robust Phase-Based Decoding for Absolute (X, Y, $\Theta$ ) Positioning by Vision
}

\author{
Antoine André, Patrick Sandoz, Benjamin Mauze, Maxime Jacquot, \\ Guillaume Laurent
}

\section{To cite this version:}

Antoine André, Patrick Sandoz, Benjamin Mauze, Maxime Jacquot, Guillaume Laurent. Robust Phase-Based Decoding for Absolute $(\mathrm{X}, \mathrm{Y}, \Theta)$ Positioning by Vision. IEEE Transactions on Instrumentation and Measurement, 2020, 70, pp.1 - 12. 10.1109/TIM.2020.3009353 . hal-03053018

\section{HAL Id: hal-03053018 https://hal.science/hal-03053018}

Submitted on 10 Dec 2020

HAL is a multi-disciplinary open access archive for the deposit and dissemination of scientific research documents, whether they are published or not. The documents may come from teaching and research institutions in France or abroad, or from public or private research centers.
L'archive ouverte pluridisciplinaire HAL, est destinée au dépôt et à la diffusion de documents scientifiques de niveau recherche, publiés ou non, émanant des établissements d'enseignement et de recherche français ou étrangers, des laboratoires publics ou privés. 


\title{
Robust phase-based decoding for absolute $(\mathrm{X}, \mathrm{Y}, \Theta)$ positioning by vision
}

\author{
Antoine N. André, Patrick Sandoz, Benjamin Mauzé, Maxime Jacquot, Guillaume J. Laurent
}

\begin{abstract}
Computer vision is a convenient non-contact tool for position control and thus constitutes an attractive multi-directional alternative to widely used single-direction sensors. However, to meet actual industry requirements, vision-based measurement methods must be sufficiently robust to comply with industrial environments. This paper explores the robustness of an in-plane position measurement method based on a pseudo-periodic pattern and allowing a $10^{8}$ range-to-resolution ratio in displacement and a $1 \mu \mathrm{rad}$ angular resolution over $2 \pi \mathrm{rad}$. The paper details how the pattern phase can be used to maintain reliable measurements despite defocus, discrepancies in local contrast, non-uniform illuminations or occlusions. Proposed method can be implemented at different size scales with unique capabilities combining high resolution, large measurement range and robustness to diverse kinds of disturbances.
\end{abstract}

\section{Index Terms}

computer vision, large range-to-resolution ratio, phase encoding, positioning, robustness

\section{INTRODUCTION}

As industrial applications advance toward ever more precise processes and manufacturing, ever more accurate positioning methods are required and, as a response, researches around sensors aim to provide ever more accurate ways to retrieve 1D or multi-dimensional positions and displacements. Currently, several techniques are commonly used to monitor position or displacement such as linear encoding [1], [2], piezoresistive sensing [3] or capacitive sensing [4], [5].

However, when the required accuracy approaches the nanometer, the number of options is reduced and the most reliable way to achieve such a precision is to rely on interferometry. Interferometers are able to detect sub-nanometer variations of distances along large ranges up to meters [6]. Interferometry is basically a one-dimensional method and multi-dimensional sensing can be achieved by multiplying the number of interfering beams at the cost of system complexity and steric hindrance [7], [8]. However, rotations remain difficult to measure by interferometric means, especially when large angular ranges are expected [9].

On the other hand, over the last decades computer vision emerged as a very convenient way to control multi degrees of freedom positions and displacements at different size scales. The combination of computer vision with optical microscopy allows accuracy to enter the sub-micrometer range and constitutes a relevant alternative to interferometers in a proportion of applications [10]-[14]. A major advantage of computer vision is to be intrinsically contactless and accuracies in the nanometer range can be demonstrated for in-plane $(x, y, \theta)$ positioning [15], [16].

Computer vision methods rely usually on images acquired in nominal conditions that optimize contrast, illumination uniformity and prevent occlusion. However, such optimal conditions are difficult to guaranty in an industrial environment and this raises the issue of the robustness of computer vision methods. This paper tackles this point by exploring the robustness against diverse types of image degradation of one computer vision method for absolute $(x, y, \theta)$ position retrieval that we introduced recently [17]. The method used relies on a pseudo-periodic pattern and combines the absolute identification of the period order with a sub-periodic interpolation to achieve a range-to-resolution ratio up to $10^{8}$. The high resolution is obtained through phase measurements that rely on the periodic frame of the pattern and benefit from the redundancy of information uniformly distributed over every recorded image. The absolute measurement is based on missing lines and columns that alter the periodic frame of the pattern and encoded by means of a LFSR binary sequence. A previous paper demonstrated the nanometer resolution permitted by this approach as well as the achievement of large range-to-resolution ratios (up to $10^{8}$ ) [17]. These nominal performances are tied to the recording of good quality images. This paper focuses on the phase-based decoding of the absolute position and aims to demonstrate the robustness of this approach in the case of low quality images (defocusing, poor contrast, uneven light and occlusion). The high level of robustness achieved results from a phase-driven decoding whose procedures are explained with full details in Sect IIII. Then, the actual robustness of the method is estimated through both experiments and simulations and the results obtained are presented and discussed.

The next section presents the principle chosen for the absolute encoding of the in-plane position $(x, y, \theta)$ within a 2D pseudo-periodic pattern in regard to related methods reported in literature. Then section III] presents the decoding procedures that constitute the algorithmic roots of the method robustness. Section IV demonstrates the method robustness through both experiments and simulations. Section $\mathrm{V}$ concludes the paper.

The authors are with the FEMTO-ST Institute, Univ. Bourgogne Franche-Comté, UMR CNRS 6174, 25000 Besançon, France \{antoine.andre, patrick.sandoz, benjamin.mauze, maxime.jacquot, guillaume.laurent\}@femto-st.fr 


\section{PATTERN-BASED POSITION ENCODING}

\section{A. Related work}

Computer-vision offers many possibilities to retrieve the position of an object. An effective way of positioning is to search for features like rectangles [18], lines [19]-[21], circles [10] or any contrasted feature steadily present in the observed scene [11]. Then, position retrieval is based on the detection and sharp localization of these features. One of the main advantages of this method is that once the feature is detected successfully, the positioning only relies on geometrical objects fitting the reality of the acquired image. As these methods rely on local detection, no interpolation greater than a fraction of a pixel can be achieved with resolutions about one tenth of a pixel. Although some degree of robustness to image alteration with noise and blur can be demonstrated [22], robustness to occlusion is weak because necessary features disappear.

Another family of computer vision methods involve some kind of image correlation to compute the relative displacements between sets of similar images. The ability of correlation techniques to achieve subpixellic resolution was demonstrated and sub-nanometer precision was proven experimentally [23]. These global methods are compliant with partial occlusion since positioning is based on the whole image [16], [24]. Studies on structured areas produced by the environment like noise [25], textures of the material [26] or diffraction caused by the lenses [27] proved their capabilities to achieve nanometric positioning. These correlation methods are highly dependent on the signal-to-noise ratio since its use is based on image information and are therefore less robust against image disturbances.

A third family of computer vision methods restricts the domain of image correlation to a narrow band of spatial frequencies by inserting periodically patterned features in the observed scenes. Then, positioning involves diverse types of Fourier-like phase computations suited to finely position the periodic image. The selection of a narrow spectral band results in efficient noise rejection and highly-subpixelic accuracies down to the nanometer range were demonstrated [15], [28], [29]. The periodic character of those phase-based methods restricts the intrinsic range of measurement to a single period of the pattern but diverse means were explored to address extended ranges. Guelpa et al. proposed a twin-grid pattern to enlarge the measurement range by a factor of about 20 whereas other authors inserted a binary code within the periodic frame [30]-[32]. A key property of encoded methods is that the measurement range is no longer defined by the field of observation of the camera but by the physical extension of the periodic pattern. This makes the measurement range independent of the vision system magnification and, combined with the highly-subpixelic resolution allowed by phase computations, large range-to-resolution ratios can be obtained [17], [33]. However, computer vision relies intrinsically on the quality of acquired images and, in industrial applications, to acquire great quality images is often difficult. The robustness of the numeric methods involved is therefore of prime importance to comply with actual application contexts. This article presents the robustness permitted by the development of specific phase-based position decoding procedures and suited for the retrieval of absolute 3-DoF positions even in the case of low quality images while preserving the high resolution and extended measurement range capabilities of the method.

\section{B. Phase-based position encoding}

Since the working principle of the proposed method is analog to laser interferometers that are considered as a reference method for displacement measurement, let us briefly review their working principle. Laser interferometers are basically 1D measurement devices that convert positions into an entire number of light wavelengths plus a sub-wavelength extra-distance: $x=k_{x} \cdot \lambda+\delta_{x}$ where $x$ is the distance to be measured, $k_{x}$ is the entire number of wavelengths (or half wavelength in the case of round trip light travels), $\lambda$ the laser wavelength and $\delta_{x}$ the residual sub-wavelength distance with $-\lambda / 2<\delta_{x} \leq \lambda / 2$. Thus, the performances of laser interferometry rely on the fact that the intrinsic measurement range is limited to a single light wavelength. In practice, $\delta_{x}$ is measured as an interferometric phase $\phi_{x}$ with $\phi_{x}=2 \pi \delta_{x} / \lambda$. The additional entire number of wavelengths $k_{x}$ is accumulated by different methods of fringe counting and this extends the measurement range beyond meters. Then the ultimate accuracy than can be achieved depends on the signal to noise ratio of the entire detection chain.

The pattern-based phase encoding method adapts interferometry principles to the context of computer vision to perform three degrees-of-freedom (DOF) measurements of in-plane position and displacements with a high accuracy. Similarly to interferometry, positions $(x, y)$ are subdivided within an entire number of wavelengths plus a sub-wavelength extra-distance. However, the wavelength is no longer provided by a propagating physical wave but by periodic patterns fixed onto the moving object of interest and observed by a vision system. The method is then thought to retrieve the in-plane coordinates $x$ and $y$ through a two-step process identifying parameters $\delta_{x}, \delta_{y}$ and $k_{x}, k_{y}$ successively. These parameters satisfy the following equations:

$$
\begin{aligned}
& x=k_{x} \cdot \lambda_{x}+\delta_{x}, \\
& y=k_{y} \cdot \lambda_{y}+\delta_{y},
\end{aligned}
$$

where $\lambda_{x}$ and $\lambda_{y}$ are the known periods of the regular features fixed along the $\mathrm{X}$ and $\mathrm{Y}$ directions of the object respectively. Furthermore, the object directions $X$ and $Y$ can be compared to the static pixel frame of the camera thus allowing the detection of the in-plane angle $\theta$ representative of object orientation. In this way, a three DOF computer vision method is obtained, 

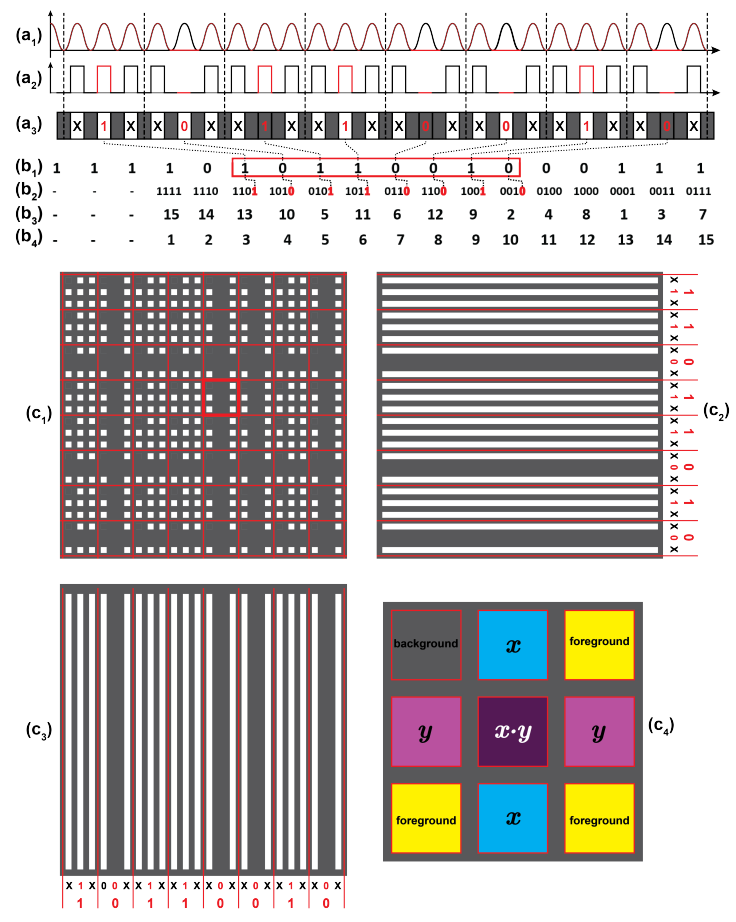

Fig. 1. Binary phase encoding process. $\left(a_{1}\right)$ Black; typical interference signal. Red; missing fringes used for binary encoding. ( $\left.a_{2}\right)$ Binary form of signal $\left(a_{1}\right)$. $\left(a_{3}\right)$ : corresponding sequence of black and white stripes. $\left(b_{1}\right)$ LFSR binary sequence with $n=4$ bits. $\left(b_{2}\right)$ : binary words formed by 4 successive bits. $\left(b_{3}\right)$ : corresponding code value. $\left(b_{4}\right)$ : corresponding linear position. $\left(c_{1}\right)$ : example of $2 \mathrm{D}$ encoded area. $\left(c_{2}\right)$ corresponding $1 \mathrm{D} x$ encoding. $\left(c_{3}\right)$ corresponding 1D $y$ encoding. $\left(c_{4}\right)$ : elementary coding cell made of $3 \times 3$ periods. The missing dot at the upper left corner aims to remove $\pi / 2$ rotation ambiguities.

equivalent of single DOF interferometer devices through the design of a suitable 2D featured pattern. The latter is required to allow the robust and accurate extraction of parameters $k_{x}, k_{y}, \delta_{x}, \delta_{y}, \theta$ from each image recorded by the vision system.

\section{Large range position encoding}

The pattern used (Fig. 1 $c_{1}$ ) is made of an altered 2D periodic distribution of dots that results from the product of 1D lines and columns (Fig. $11 c_{2}-c_{3}$ ). The periodic frame is aimed to allow accurate measurements of $\delta_{x}$ and $\delta_{y}$ through phase measurements whereas alterations produced by the missing lines and columns encrypt a binary code ensuring the unambiguous identification of the line and column orders as necessary to identify parameters $k_{x}$ and $k_{y}$. Thus $x$ and $y$ are obtained through phase measurements with: $\Phi_{x}=2 k_{x} \pi+\phi_{x}$ and $\Phi_{y}=2 k_{y} \pi+\phi_{y}$ where $\phi_{x}=2 \pi \delta_{x} / \lambda_{x}$ and $\phi_{y}=2 \pi \delta_{y} / \lambda_{y}$. The binary code encryption method has been chosen in order to fulfil three complementary requirements: $i$ ) to avoid the introduction of non-linear distortions of the periodic distribution of lines and columns; ii) to provide information redundancy beneficial to decoding robustness; and iii) to relate encoding features to phase information. For that purpose, binary values 1 and 0 are represented by sets of three periods and distinguished by the central period that is present when the bit is equal to 1 and absent otherwise (Fig. 1 a). In this way, the binary code reduces the magnitude of the periodic frame spectrum but does not alter its phase that encodes the position of the lines and columns with respect to the image pixel frame. This point constitutes a crucial improvement in regard to several encoding methods proposed earlier and inducing non-linearities [34], [35].

The encryption of the line and column orders is based on linear feedback shift register sequences that require a single binary track whatever the number of bits considered (Fig. 1]b) [34], [36]. In this encoding scheme, each word of $n$ bits shares $n-1$ bits with its immediate neighbors and the absolute position can be retrieved from any sequence of $n$ consecutive bits as depicted in Fig. 1 $\mathrm{b}$ for $n=4$. Combined with the choice to encode each bit with three periods, this technique allows the encoding of a spatial length equal to $3 \cdot\left(2^{n}-1\right)$ periods from a sequence of $3 \cdot\left(2^{n}+n-2\right)$ bits whereas only $3 n$ consecutive periods are required for the decoding of the current position. Therefore, the ratio between the measurement range and the minimum field of observation is given by $\left(2^{n}-1\right) / n$ and increases with $n$. Then the proper choice of the effective values of the physical periods $\lambda_{x}$ and $\lambda_{y}$ and of the bit number $n$ allows the design of encrypted patterns suitable to many application requirements. Fig. 1 summarizes the encoding procedure from the differentiation of bit values (Fig. 1 a), to the encryption of position by means of LFSR sequences (Fig. 1 b), to end with the resulting 2D pattern (Fig. 1 $\mathrm{c}$ ). Thus, any 2D position of coordinate $(x, y)$ is represented by a coding cell (Fig. 1 $1, c_{4}$ ) made of $3 \times 3$ dots of which central lines and column encode the values of $x$ and $y$ respectively. The four corners remain the same allover the 2D pattern with one missing corner to avoid $\pi / 2$ ambiguities in the in-plane angle. In this way, every coding cell provides the local values of both foreground and background intensities suitable for a robust identification of the values taken by $x$ and $y$ coordinates respectively. 


\section{ROBUST RETRIEVAL OF ABSOLUTE POSITION}

Position retrieval is performed through multiple successive steps that combine linear and Boolean processing. First linear phase computation is applied to each image of the pattern recorded by the imaging system (Fig. 2 $a$ ). This linear step interpolates the position of the pattern lines and columns with respect to the image pixel frame and this results in the high resolution of the method. The Boolean processing is aimed to identify the correct line and column orders and to remove $\pi / 2$ ambiguities on the pattern orientation. This Boolean step involves four successive steps that make best use of the phase data to maximize the overall position decoding robustness. These successive steps are explained in details in the following sub-sections.

\section{A. Linear phase processing}

To retrieve the linear phase of the pattern, a 2D Fourier transform is firstly performed on the acquired image (Fig. 2 $a$ )).

$$
F(u, v)=\sum_{i=0}^{m} \sum_{j=0}^{n} I(i, j) e^{-i 2 \pi\left(\frac{u \cdot i}{m}+\frac{v \cdot j}{n}\right)}
$$

with $I(i, j)$ the pixel intensity at the position $(i, j)$ of a $m$-columns and $n$-rows image. Resulting power spectrum is made of a set of spectral lobes and each one is associated to a particular spatial frequency (Fig. 2 $b$ ). This spectrum is filtered twice by applying Gaussian band-pass filters centered on frequencies $f_{1}$ and $f_{2}$ respectively and corresponding to the $x, y$ directions of the pattern (Fig. $2 a$ ). Since the complex conjugates of lobes $f_{1}$ and $f_{2}$ are excluded from the filter, after inverse Fourier transform, complex data are obtained of which angles give directly the wrapped phase distribution associated to the pattern lines and columns respectively (Fig. $2\left(c_{1}-c_{2}\right)$.

Since the lines and columns of the pattern are known to be regular and consecutive, the wrapped phase of Fig. $2 . c_{1}-c_{2}$ can be unwrapped to result in continuous phase planes as depicted in Fig. $2 d_{1}-d_{2}$. These phase planes can be expressed by: $\Phi_{1}(i, j)=a_{1} \cdot i+b_{1} \cdot j+c_{1}$ and $\Phi_{2}(i, j)=a_{2} \cdot i+b_{2} \cdot j+c_{2}$ where $\mathrm{i}$ and $\mathrm{j}$ are the pixel coordinates counted from the image center. In practice, coefficients $a_{1}, b_{1}, c_{1}$ and $a_{2}, b_{2}, c_{2}$ are determined by least square fitting of corresponding unwrapped phase planes (Fig. $2 d_{1}$ and $2 d_{2}$ respectively). The least square identification of these parameters allows the accurate determination of phases $\Phi_{1}(0,0), \Phi_{2}(0,0)$ that, modulo $2 \pi$, correspond to the expected phases $\phi_{x}, \phi_{y}$ of the pattern point imaged exactly at the center of the recorded image. The choice of the least square method constitutes an efficient and appropriate way to include a large number of pixels in this computation and thus to benefit from the redundancy of information spread across the unwrapped phase maps.

Finally, the pattern orientation is given by:

$$
\begin{aligned}
& \theta=\tan ^{-1}\left(\frac{b_{1}}{a_{1}}\right)+\frac{q_{1} \cdot \pi}{2} \text { or } \\
& \theta=\tan ^{-1}\left(\frac{b_{2}}{a_{2}}\right)+\frac{q_{2} \cdot \pi}{2}
\end{aligned}
$$

where integers $q_{1}$ and $q_{2}$ vary between 0 and 3 and identify the angular quadrant allowing the unambiguous determination of the pattern angle. However, the correct association between $\Phi_{1}(0,0)$ and $\Phi_{x}, \Phi_{2}(0,0)$ and $\phi_{y}$ or between $\Phi_{1}(0,0)$ and $\phi_{y}, \Phi_{2}(0,0)$ and $\phi_{x}$ remains unknown at this stage and will be determined later by removing $\pi / 2$ ambiguities on $\theta$. The determination of the angular quadrant is presented in the following section.

\section{B. Binary code retrieval}

1) Phase-based localization of relevant sites: In this step, data obtained by linear phase processing is used to localize the different encoding features of interest. In Fig. $2 d_{1}$, the red bar identifies the pixels where the phase constant is the same, i.e. verifying $2 k_{1} \pi-\pi<\Phi(i, j) \leqslant 2 k_{1} \pi+\pi$ (with $k_{1}=22$ in the presented case). The red bar was reported on the same pixels of Fig. 2 $c_{1}$ and $2 a$ thus highlighting that it corresponds indeed to a single raw of dots in the pattern image. Therefore, two intermediary images with constants can be considered: $K_{1}(i, j)=\operatorname{round}\left(\Phi_{1}(i, j) / 2 \pi\right)$ and $K_{2}(i, j)=\operatorname{round}\left(\Phi_{2}(i, j) / 2 \pi\right)$. In these images, every pattern line corresponds to a single constant $k_{1}$ in $K_{1}$ and every pattern column corresponds to a single constant $k_{2}$ in $K_{2}$.

Phase data is also used to identify the localization of the center of the dots with respect to the surrounding background as depicted in Fig. 3. For a pattern image (Fig. 3. $a$ ), pixels where both $\left|\phi_{1}(i, j)\right|$ and $\left|\phi_{2}(i, j)\right|$ are smaller than $\pi / 3$ can be extracted:

$$
B_{f}(i, j)=\left\{\begin{array}{cc}
1 & \text { if } \\
0 & \text { else }
\end{array}\left|\phi_{1}(i, j)\right|<\frac{\pi}{3} \text { and }\left|\phi_{2}(i, j)\right|<\frac{\pi}{3}\right.
$$

The Boolean image $B_{f}(i, j)$ obtained with above equation depicted in Fig. $3 . b$, localizes the sites of dot centers. Similarly, background is identified as pixels where either $\left|\phi_{1}(i, j)\right|$ or $\left|\phi_{2}(i, j)\right|$ are larger than $3 \pi / 4$.

$$
B_{b}(i, j)=\left\{\begin{array}{cc}
1 & \text { if } \\
0 & \text { else }
\end{array}\left|\phi_{1}(i, j)\right| \geq \frac{3 \pi}{4} \text { or }\left|\phi_{2}(i, j)\right| \geq \frac{3 \pi}{4}\right.
$$



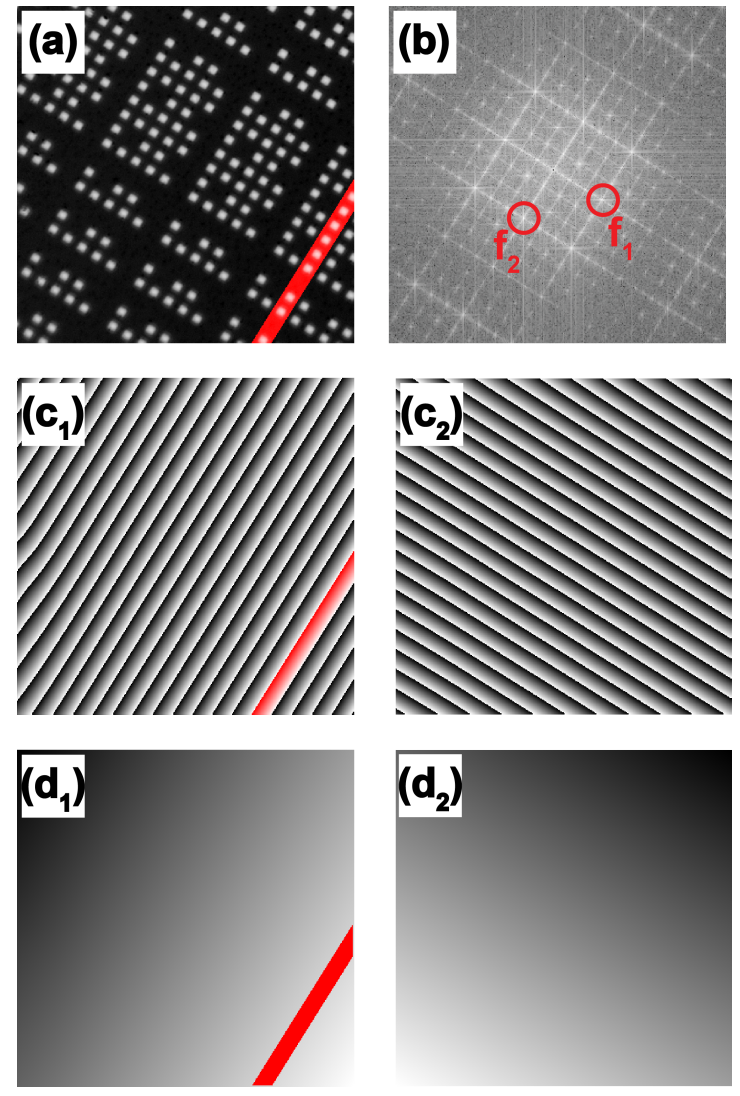

Fig. 2. Linear phase processing of a pseudo-periodic pattern. (a) grabbed image of the pattern, $(b)$ modulus of the $2 \mathrm{D}$ Fourier transform, $\left(c_{1}\right),\left(c_{2}\right)$ wrapped phase of the inverse Fourier Transform along each direction, $\left(d_{1}\right),\left(d_{2}\right)$ phase planes for both directions.

The complementary Boolean image $B_{b}(i, j)$ localizes background sites with certainty as depicted in Fig. 3 c. The RGB combination of these images (Fig. 3 d) demonstrates the ability of these thresholds to discriminate between dot centers and surrounding background. In the latter image, the combination of red and green colors gives a yellow shade to present dot centers whereas missing dot centers remain green. The appropriate choice of threshold values allows the rejection of intermediate positions that keep their initial black or dark red shades (especially visible in the right bottom corner zoom of Fig. 3 d). In these images, every green/yellow dot corresponds to the intersection of a single pattern line with a single pattern column and is therefore associated to an unique couple of phase constants $\left(k_{1}, k_{2}\right)$ as stored in images $K_{1}$ and $K_{2}$.

2) Shift toward thumbnail images: This phase-based processing allows the shift from initial images in pixels (Fig. 4 $a$ ) to thumbnail images defined by lines and columns orders $k_{1}, k_{2}$ (Fig. $4 . b$ ) that are independent of pattern orientation $\theta$ and are much smaller in size than images in pixels. The white arrows indicate the directional correspondence between Fig. $4 a$ and $4 b$. Each element $\left(k_{1}, k_{2}\right)$ of the thumbnail image gathers all image pixels of which phase constants are equal to $k_{1}, k_{2}$. In a first step, Boolean images $B_{f}$ and $B_{b}$ are used to construct complementary foreground and background thumbnail images, respectively $T_{f}$ and $T_{b}$, as well as supplementary thumbnail images $N_{f}$ and $N_{b}$ that store the number of pixels aggregated in each thumbnail image element. These four thumbnail images are defined by the following equations:

$$
\begin{gathered}
T_{f}\left(k_{1}, k_{2}\right)=\sum_{i=1}^{m} \sum_{j=1}^{n}\left(K_{1}(i, j)=k_{1}\right) \cdot\left(K_{2}(i, j)=k_{2}\right) \cdot\left(B_{f}(i, j)\right) \cdot I(i, j) \\
T_{b}\left(k_{1}, k_{2}\right)=\sum_{i=1}^{m} \sum_{j=1}^{n}\left(K_{1}(i, j)=k_{1}\right) \cdot\left(K_{2}(i, j)=k_{2}\right) \cdot\left(B_{b}(i, j)\right) \cdot I(i, j) \\
N_{f}\left(k_{1}, k_{2}\right)=\sum_{i=1}^{m} \sum_{j=1}^{n}\left(K_{1}(i, j)=k_{1}\right) \cdot\left(K_{2}(i, j)=k_{2}\right) \cdot\left(B_{f}(i, j)\right) \\
N_{b}\left(k_{1}, k_{2}\right)=\sum_{i=1}^{m} \sum_{j=1}^{n}\left(K_{1}(i, j)=k_{1}\right) \cdot\left(K_{2}(i, j)=k_{2}\right) \cdot\left(B_{b}(i, j)\right)
\end{gathered}
$$



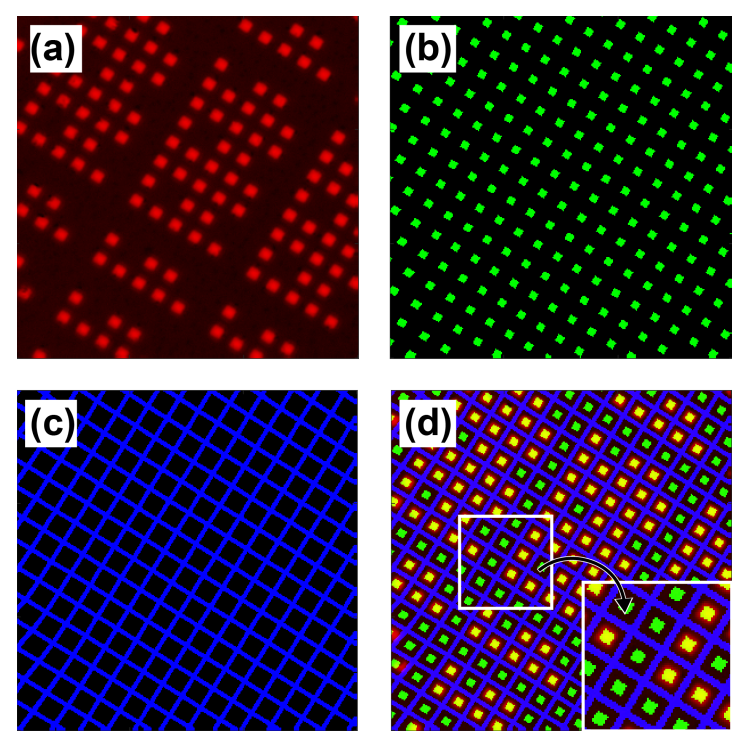

Fig. 3. Relevant site extraction. (a) grabbed image of the pattern in red, $(b)$ center of each dot site in green, (c) background of each line and column in blue, $(d)$ RGB assembly of the three previous figures $(a, b$ and $c)$.

These thumbnail images allow, for each couple of phase constant $k_{1}, k_{2}$, the computation of the mean foreground and background intensities $\bar{T}_{f}$ and $\bar{T}_{b}$ representative of the averaged intensities observed at the dot center sites and at the dot surrounding sites respectively:

$$
\begin{gathered}
\bar{T}_{f}\left(k_{1}, k_{2}\right)=T_{f}\left(k_{1}, k_{2}\right) / N_{f}\left(k_{1}, k_{2}\right) \\
\bar{T}_{b}\left(k_{1}, k_{2}\right)=T_{b}\left(k_{1}, k_{2}\right) / N_{b}\left(k_{1}, k_{2}\right)
\end{gathered}
$$

$\bar{T}_{f}$ and $\bar{T}_{b}$ are expected to take similar values for phase constants where dots are absent whereas they are expected to take significantly different values where dots are present. This is quantified by performing the element by element ratio of these thumbnail images as represented in Fig. $4 ., b$ :

$$
r\left(k_{1}, k_{2}\right)=\bar{T}_{f}\left(k_{1}, k_{2}\right) / \bar{T}_{b}\left(k_{1}, k_{2}\right)
$$

As expected and observed in Fig. 4. $b$, this ratio is close to one for absent dots whereas it varies between 6 and 12 for present dots. This ratio thumbnail image allows a robust discrimination of present and absent dots despite the increase from 6 to 12 of the ratio observed for present dots that results from non-uniform illumination. Typically, recorded images contain around 40 lines and columns spread over $1024 \times 1024$ pixels. The shift towards thumbnail images reduces thus memory requirements and computation time by involving matrices of $40 \times 40$ elements instead of images of $1024 \times 1024$ pixels. In this way, the computed set of thumbnail images provide all information necessary to perform the remaining decoding steps.

3) Code synchronization: A clear discrimination between present and absent dots appears in the thumbnail images of Fig. $4 . b$. The regular distribution of missing dots designed to remove $\pi / 2$ angular ambiguities appears also clearly every three phase constants in both directions.

The aim of this step is the robust determination of the phase constants modulo 3 that correspond to the coding lines and columns as well as the phase constants associated to the always missing dot. This is performed by computing a $3 \times 3$ global cell $\bar{G}(u, v)$ that aggregates modulo 3 the thumbnail image representative of the dot center site intensities as follows:

$$
\begin{gathered}
G(u, v)=\sum_{k_{1}} \sum_{k_{2}} T_{f}\left(k_{1}, k_{2}\right) \cdot\left(k_{1} \equiv u\right) \cdot\left(k_{2} \equiv v\right) \\
N_{G}(u, v)=\sum_{k_{1}} \sum_{k_{2}} N_{f}\left(k_{1}, k_{2}\right) \cdot\left(k_{1} \equiv u\right) \cdot\left(k_{2} \equiv v\right) \\
\bar{G}(u, v)=G(u, v) / N_{G}(u, v)
\end{gathered}
$$

with $u$ and $v$ varying between 1 and 3. The global cell obtained is represented in Fig. $4 . c$ and is interpreted as follows: The minimum intensity (violet at the top left corner) corresponds to the mean center intensity of always missing dots and is easily localized. This minimum determines which phase constants modulo 3 are associated with always missing dots. This allows the determination of the angular quadrant and leads to the absolute pattern orientation presented in equation eq. 3 . Similarly, the three maximal values (yellow) correspond to always present dots that are also easily identified as well as corresponding phase 

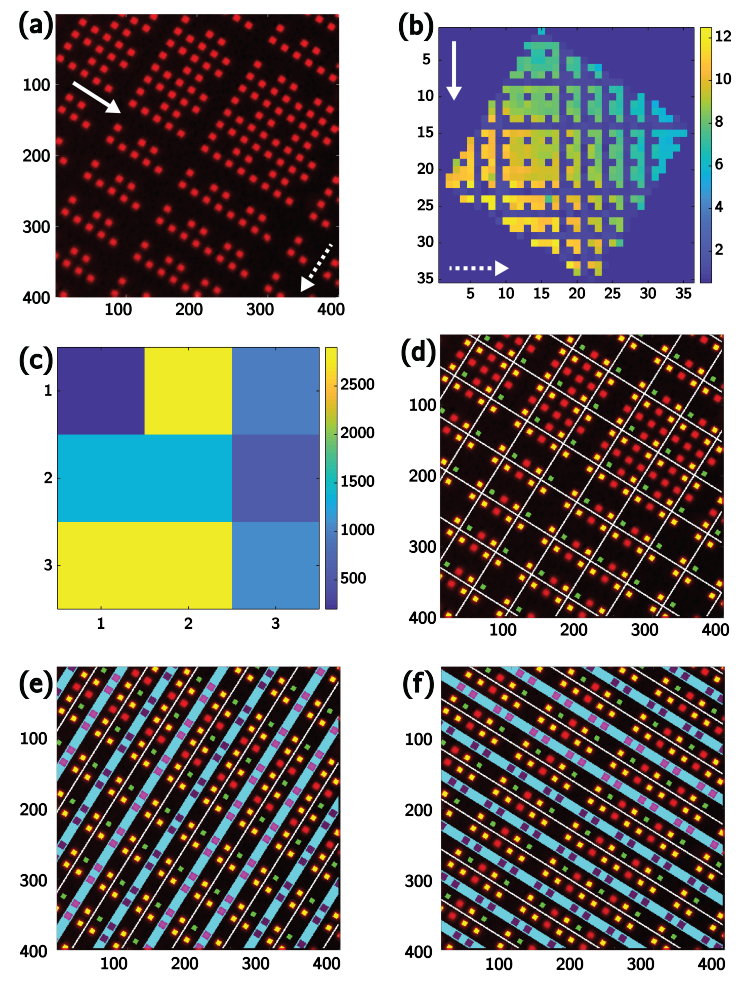

Fig. 4. Thumbnail processing: $(a)$ Pattern image in pixels; $(b)$ Thumbnail image of the ratio between foreground and surrounding background (versus phase constants); $(c)$ Global cell obtained; $(d)$ Pattern image with coding cell borders and invariant dots; $(e)$ Binary decoding versus first direction; $(f)$ Binary decoding versus second direction. Magenta color marks dots actually considered for binary value determination $($ bright $=1$; dark $=0)$.

constants modulo 3. Among the remaining points, two (cyan, blue) duets correspond to coding cell positions that encode for $x$ and $y$ values respectively (or $y$ and $x$ values) whereas the last point (middle of right column), with an intensity lower than that of the duets, corresponds to the coding cell center determined by $x \cdot y$. This intensity classification of the nine global cell elements allows thumbnail image synchronization; i.e. the unambiguous identification of the distribution of the coding cells and of the always present and always missing dots with respect to actual phase constants.

This synchronization specific to every processed image is then used as depicted in Fig. $4 . d$ - $f$. In Fig. $4 . d$, the white lines mark the identified contours of the coding cells whereas yellow and green dots correspond to always present and always missing dots respectively. Fig. 4 . $e$ depicts the determination of the binary value for the first direction. The coding lines are highlighted in cyan and their binary values are determined by comparing the averaged intensity of dots encoding only for this direction (dark or bright magenta) to surrounding background. At this stage, dots encoding for both directions are voluntary discarded to avoid disturbances introduced by the perpendicular direction. Furthermore, all dots included within two consecutive white lines are considered for the determination of the corresponding binary value and with a weight proportional to the number of pixels of each dot. Fig. $4 . f$ presents the same process for the second direction.

The aim of Fig. 4. $d-f$ is to explain clearly the principle and robustness of the phase-based binary decoding. However, the actual process is carried out from thumbnail images that concentrate the same information within smaller matrices that save computation resources. Furthermore, for the sake of readability, Fig. 3 and 4 show only a fraction of the recorded pattern images. Once applied to the whole pattern image, this binary processing provides the phase constants associated to the coding lines and columns as well as to the sites of always present and always absent dot centers. This knowledge allows a local and robust identification of the binary sequence as explained below.

4) Final binary sequence identification: The final identification of the binary sequence makes use of the phase processing described previously as depicted in Fig. 5. For each set of three line and column phase constants, the mean foreground and background intensities are computed as explained in Fig. 4. $e$ - $f$ by computing the averaged intensity of pixels located at the center of always present and always absent dots respectively. In Fig. 5 $a_{1}$ and $5 a_{2}$, for each set of three lines and columns, the mean foreground intensity of always present dots is represented by a yellow bar whereas that of always missing dots is represented by a green bar. These intensity values serve as local references to evaluate the correct binary value of corresponding coding lines and columns represented by bars in magenta. The length of the latter is proportional to the mean foreground intensity of dots coding only for that direction (appearing in light or dark magenta in Fig. 4 . $e-f$ ). Fig. $5 a_{1}$ and $5 a_{2}$ show clearly that the mean foreground intensity of coding dots is either stuck to always present dots or to always missing 


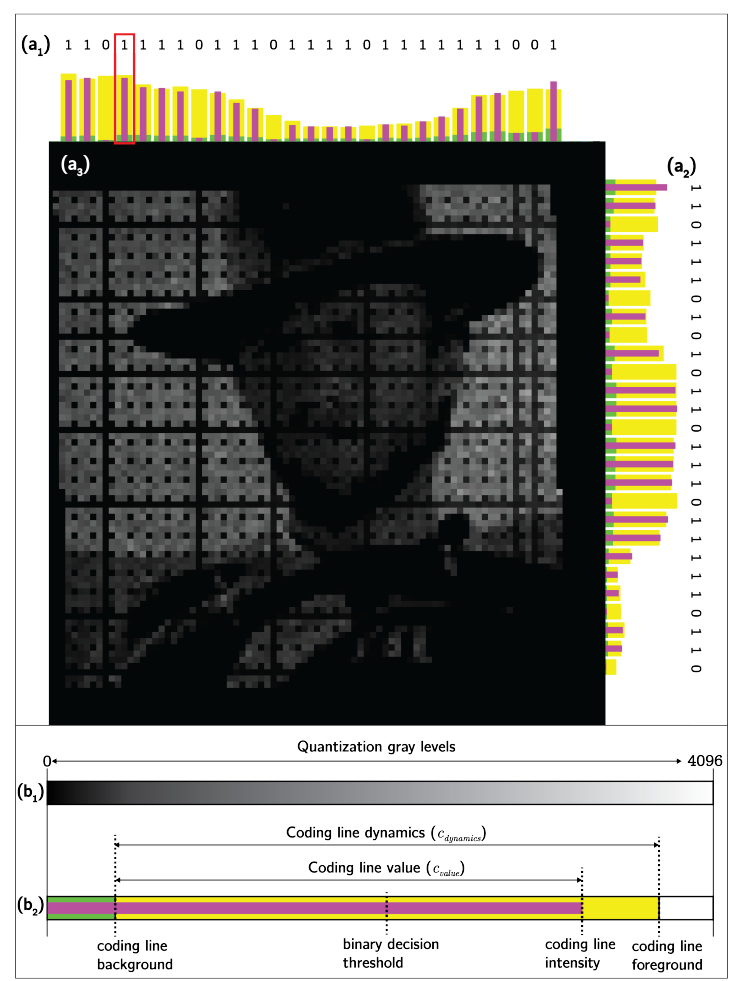

Fig. 5. Binary value determination from the thumbnail foreground intensity image. $\left(a_{1}\right)$ thumbnail of an image altered by white noise, occlusion and variable light (extracted from supplemental material 1.). For each set of three lines and columns, bars in $\left(a_{2}\right)$ and $\left(a_{3}\right)$ compare the mean coding intensity (magenta) to the foreground (yellow) and background (green) mean intensities with the determined binary value indicated beside. $\left(b_{1}\right)$ shows the dynamics allowed by the 12 bits quantification. $\left(b_{2}\right)$ depicts the decision criterion for the coding column within the red rectangle in $\left(a_{1}\right)$ with respect to the 4096 gray level range.

dots, even in the case of a significantly altered image as chosen in Fig. $5 a_{3}$. Mathematically, the binary value of each coding line $k$ is determined with the use of a coding ratio $\left(c_{r}\right)$, defined with the following equation in accordance with Fig. $5 b_{2}$ :

$$
c_{r_{i}}=c_{\text {value }}\left(k_{i}\right) / c_{\text {dynamics }}\left(k_{i}\right)
$$

with $i=0$ or $i=1$ representing the considered coding vector direction. The ratio $c_{r}$ is equal to 0 when the coding line intensity is equal to the background line intensity and equal to 1 when the coding line intensity is equal to the foreground line intensity. This allows to determine each binary value of the coding vector $V_{1}$ (and $V_{2}$ for the other direction) which is an extract of the LFSR sequence presented in section $[\mathrm{II}-\mathrm{C}$

$$
V_{i}\left(k_{i}\right)=\left\{\begin{array}{lll}
0 & \text { if } & c_{r_{i}} \leq 0.5 \\
1 & \text { if } & c_{r_{i}}>0.5
\end{array}\right.
$$

As represented in Fig. $5 b_{2}$ this criterion of binary value determination leads to keep the decision threshold at the middle of the background and foreground intensities whatever their values in regard of the camera quantification range (Fig. $\left.5 b_{1}\right)$. Finally, determining the position of $V_{1}$ and $V_{2}$ in the LFSR sequence provides to the phase constants $k_{x}$ and $k_{y}$. In this way, the absolute position of the phase-encoded pattern which is imaged exactly at the central pixel of the camera is finally obtained with respect to eq. 11. Supplemental material 2. summarizes the presented steps of the binary decoding process in the case of a rotation.

\section{RoBUSTnESS ANALYSIS}

In order to investigate the robustness of the proposed method, both experimental and simulated studies were put in effort. The following sub-sections describe these tests and present the obtained results.

\section{A. Experimental setup and nominal performances}

A test of maximal performances under nominal conditions is firstly made and will serve for further robustness results as a reference tool. To estimate these performances, a resolution test including a static noise study and a minimal movement detection is put in practice. This experiment is made in a room on an independent basement (for vibration limitation) and on an anti-vibration table. The room is regulated in temperature $\left(22.4^{\circ} \mathrm{C}\right)$ to minimize thermal drifts and its pressure is controlled at $8 \mathrm{~Pa}$ above the external pressure to keep the airflow toward the outside and thus reduce the entrance of external dust. An 


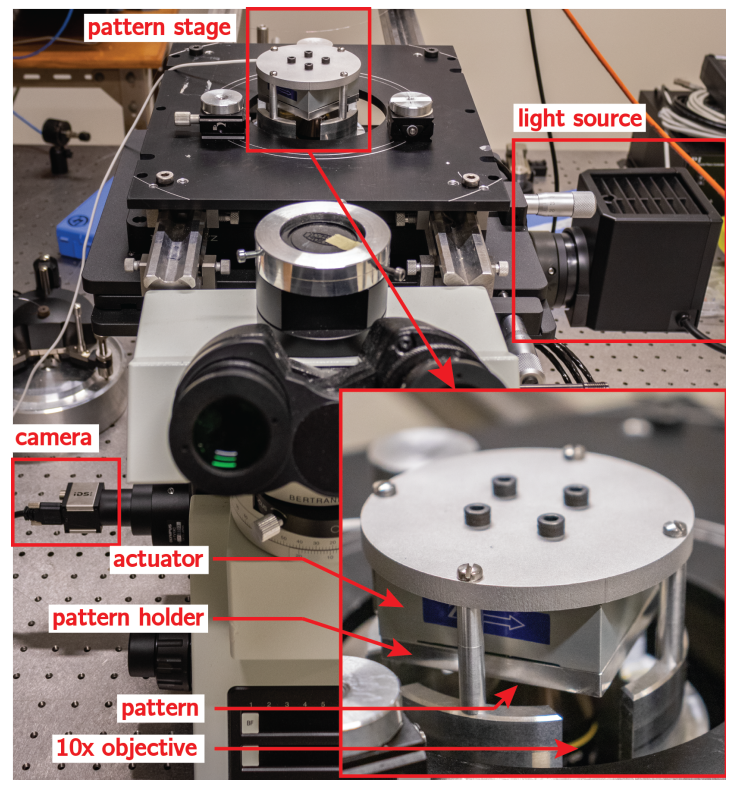

Fig. 6. Experimental setup showing the inverted microscope used and the actuation stage holding the encoded periodic pattern.

TABLE I

EXPERIMENTAL STD POSITION OVER THE THREE DOFS OF THE PATTERN IN A STATIC EXPERIMENT ACQUIRED ON A 20 SECONDS SET WITH 20 FRAMES PER SECOND ACQUISITION. IN LOW LIGHT DYNAMIC CONDITIONS THE STD IS DOWNGRADED BY A FACTOR 26 IN TRANSLATION ( $x$ AND $y$ DIRECTIONS) AND APPROXIMATELY BY 100 IN ROTATION.

\begin{tabular}{|c|c|c|c|c|}
\hline & $\begin{array}{c}\text { Image } \\
\text { dynamics }\end{array}$ & $\begin{array}{c}\text { STD along } \\
x \text { axis }\end{array}$ & $\begin{array}{c}\text { STD along } \\
y \text { axis }\end{array}$ & $\begin{array}{c}\text { STD of } \\
\theta \text { angle }\end{array}$ \\
\hline $\begin{array}{c}\text { Nominal } \\
\text { conditions }\end{array}$ & $0-3754$ & $0.57 \mathrm{~nm}$ & $0.51 \mathrm{~nm}$ & $1.43 \mu \mathrm{rad}$. \\
\hline $\begin{array}{c}\text { Low light } \\
\text { conditions }\end{array}$ & $70-90$ & $13.29 \mathrm{~nm}$ & $13.85 \mathrm{~nm}$ & $99.84 \mu \mathrm{rad}$. \\
\hline
\end{tabular}

inverted microscope (Olympus IX73) with a 10× lens objective (Olympus UPLFLN 10x, N.A. 0.30) is used for the optical imaging. An adapted table stage was made to support the actuator (MCL Nano-MET10 with $0.01 \mathrm{~nm}$ resolution) where the encoded pattern is fixed underneath. The camera used for acquisition is an IDS (UI-3280CP-M-GL) set at 20 frames per second encoded on 12 bits (allowing 4096 levels of gray). Fig. 6 summarizes the implementation of the experiment. An exposure of $10.5 \mathrm{~ms}$ is chosen as it reveals the highest lighting dynamics to acquire the pattern images (Fig. $7 a_{1}$ ).

The pattern used is designed with 12 bits encoding accordingly to the method presented in part II-A with a $9 \mu$ m physical period. With these parameters, the pattern is $11 \times 11 \mathrm{~cm}^{2}$ wide. It was realized in clean room with a commercial mask generator (Heidelberg DWL200) on a $12.5 \times 12.5 \mathrm{~cm}^{2}$ piece of glass of $3 \mathrm{~mm}$ thickness. For practical reasons, only a $2 \times 2 \mathrm{~cm}^{2}$ fragment is used in the described experiment. A sample of the acquired pattern image is presented in Fig. $7 . a_{1}$ were a few coding lines are visible.

In nominal conditions of image acquisition (i.e. no occlusions, widest image dynamics and maximal contrast), a noise level below one nanometer for each translation axis and around one microradian along the rotation axis can be demonstrated. Results values are summarized in Table [] Furthermore, a resolution test is made to demonstrate the lowest incremental movement that can be detected. This test is carried only in one direction since the actuator is unidirectional. To proceed, a $1 \mathrm{~nm}$ amplitude square signal at $0.5 \mathrm{~Hz}$ is sent to the actuator. The experiment proved to detect these movements as shown in Fig. $7 a_{3}$. As demonstrated in a previous article [17], with these experimental conditions, the pattern can be used to position a frame in a range of $11 \mathrm{~cm}$ with a sub-nanometric resolution and thus allowing a range-to-resolution coefficient of $10^{8}$. Efficiency in terms of time cost of the process was quantified during the tests presented throughout the paper. The positioning algorithm programmed in $\mathrm{C}++$ has a $O(n \log (n))$ complexity and allows real time measurements. Table $\Pi$ shows the computation time needed to process the measure in regard to the input image size. Parallel processing could be implemented to shorten the computing time further. 
TABLE II

COMPUTATION TIME VERSUS INPUT IMAGE SIZE

\begin{tabular}{|c|c|}
\hline Image size & Computation time $(\mathrm{ms})$ \\
\hline $256 \times 256$ & 7.70 \\
\hline $512 \times 512$ & 33.07 \\
\hline $1024 \times 1024$ & 148.53 \\
\hline
\end{tabular}

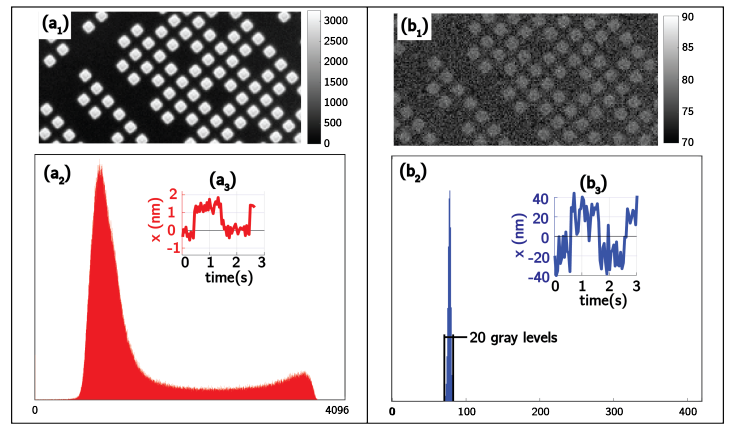

Fig. 7. Resolution robustness comparison in different lighting conditions. $\left(a_{1}\right)$ is a zoom on pattern image acquired in high light dynamics and $\left(b_{2}\right)$ is a zoom on pattern image with the lowest available lighting conditions. $\left(a_{2}\right)$ histogram of the pattern image, revealing a bi-modal shape stretched out on the 12 bits of gray levels quantization. $\left(b_{2}\right)$ histogram shows that all the values of the pattern image are packed between 70 and 90 gray levels (the $\left(b_{1}\right)$ image is mapped accordingly to these gray levels). $\left(a_{3}\right)$ and $\left(b_{3}\right)$ show resolution tests and reveal that in favorable conditions, a sub-nanometric resolution can be achieved. In low-light conditions, the tiniest detectable movement is $40 \mathrm{~nm}$.

\section{B. Robustness against low light conditions}

To demonstrate the robustness to poor lighting environment, the light source is set to a minimal value and the exposure time decreased consequently, going from $10.5 \mathrm{~ms}$ to $0.5 \mathrm{~ms}$. The resulting images are hardly possible to decode by visual checking. To ensure they aren't totally black, histograms are used to reveal the maximal image intensity value. As shown in Fig. $7 b_{2}$, the pattern presents a maximal intensity of 90 out of 4096 values allowed by the 12 bits image encoding. With a normalization of the acquired image between 70 and 90 levels of gray, the pattern is visually unveiled as shown in Fig. $7 . b_{1}$. In these peculiar conditions, the same experimental protocol as for the nominal test is conducted.

Position analysis shows that even at low light level (20 levels of gray), the pattern is still detected and well positioned. Since the method is based on a spectral analysis, light dynamics doesn't interfere with the Fourier transform and a frequency peak can still be detected, allowing the described phase-based positioning method to work. By recording the noise level in static conditions, results show an increase from $0.5 \mathrm{~nm}$ in nominal conditions to $13 \mathrm{~nm}$ in low light conditions (Tab. I). Resolution test in low light dynamics shows that a step of $40 \mathrm{~nm}$ can be detected (Fig. $7 . b_{3}$ ).

\section{Robustness against defocus}

Combined with a microscope, computer-vision is generally limited by the short depth of field of microscope objectives. In the case of the conducted experiment, a Mitutoyo 10× Plan apo (N.A. 0.28) with a depth of field of $3.5 \mu \mathrm{m}$ is used. The goal of this study is to highlight the robustness of the decoding method against defocus. The encoded pattern presented in the previous part is placed on a 6DOFs robot assembled from SmartAct nanopositioning stages (more details in [17], [37]). The pattern is translated along the optical axis on a range of $180 \mu \mathrm{m}$ and images are acquired every $3 \mu \mathrm{m}$. The overall evolution of the pattern during the experiment can be seen in supplemental material 3 .

The focus measure of acquired images is evaluated by using the method presented by Krotkov [38]. This method based on the gray-level variance uses the following equation that is widely used [39]:

$$
\sigma^{2}=\frac{1}{m \cdot n} \sum_{i=1}^{m} \sum_{j=1}^{n}(I(i, j)-\mu)^{2}
$$

where $\mu$ stands for the mean of the image. As the image becomes more and more in focus, the grey-level difference between adjacent pixels grows and the value of $\sigma$ increases. The resulting normalized curve of focus measure is presented in the Fig. 8 Experimentally, the position is found to be correctly reconstructed over an axial distance of $120 \mu \mathrm{m}$ (Fig. 8), thus showing the compliance of the method against defocus. In the case studied and for the purpose of positioning, the objective depth-of-field is extended by more than 30 times. This proportion factor depends on diverse experimental factors among which the value of the pattern spatial frequency relatively to the cut-off frequency of the modulation transfer function of the objective plays a predominant role. 


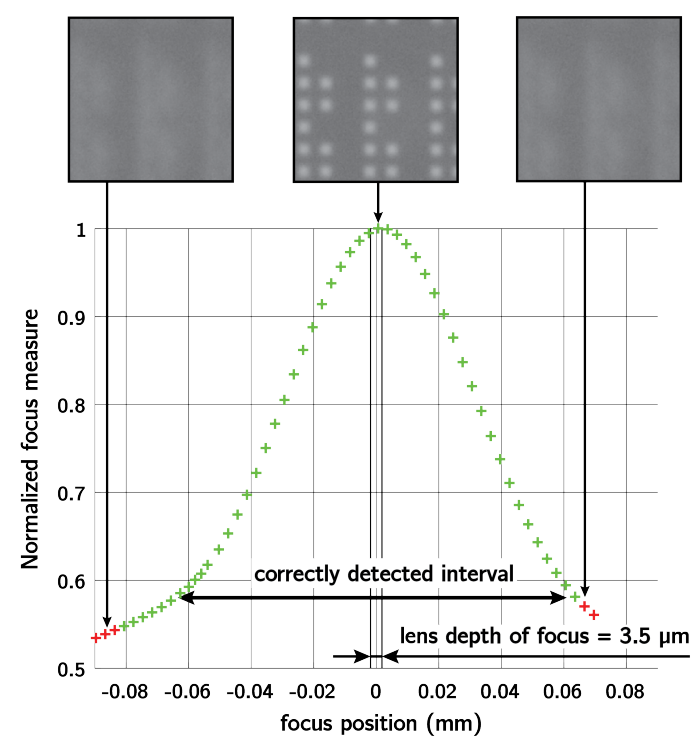

Fig. 8. Pattern image focus measure for a focus swept over $180 \mu \mathrm{m}$. Correctly decoded pattern images appear in green while badly decoded ones appear in red. Samples of pattern images over the focus curve show the conditions of decoding at the extremities of the curve and at the ideal focus point.

\section{Robustness against diverse types of noise}

Simulations were carried out to explore the robustness of the method against three types of image alteration; namely addition of a non-uniform light background, progressive addition of white noise, blending occlusion with a movie. The protocol is set as follow : a $1024 \times 1024$ pattern image is generated at a random position. Then the coded sequence appearing in both directions at this position is recorded and is used as a reference. Various alterations are progressively applied to the image analyzed by the positioning method, generating 100 resulting degraded images for each presented case. Evolution of the pattern image degradation is available as a short clip presented in supplemental material 1. During the decoding process, information shown in Fig. $5 . b_{2}$ are recorded so they will be used for the robustness studying.

Every coding ratio (as defined in section III-B4 coming from every line is recorded and stored. Fig. 9 summarizes these values for every degraded image analyzed by the process. In order to make the result more visual, coding ratio values are placed according to the corresponding coding line lighting dynamics (calculated as $\left(\bar{I}_{\text {f oreground }}-\bar{I}_{\text {background }}\right)$ /4096). Results show the binary value (presented in equation eq. 177) computed either in green or in magenta depending on the validity of this one (by comparing the reference line binary value computed without alteration with the one computed after image degradation). Over the 14500 binary values compared, only 43 revealed to be badly binarized. Furthermore, these binarization errors mainly occur in poor light dynamics (below 0.05) and when few pixels are involved in the binarization process. This proves that the image positioning complies with very poor lighting dynamics and high noising effects and still perform well the decoding under peculiar acquisition conditions. Furthermore, depending on the image size, the decoded binary sequence can be larger than required by the word depth of the LFSR sequence. In such cases, the correct position can still be expected even if one bit on an image side was attributed the wrong value. For instance, despite the 43 errors counted in Fig. 9, the full image sequence was successfully localized at the expected positions among the pattern area.

\section{E. Robustness against occlusion}

In the case of stain or deterioration of the physical pattern, the acquired pattern image becomes less and less readable as the rate of occlusion increases. The method robustness against occlusion was studied through simulations based on images of $1024 \times 1024$ pixels with random position and orientation distributed among the whole pattern area. Then the image is progressively occluded by black circles with a radius randomly distributed between 20 and 75 pixels and localized at a random position in the image. The process is then continued until $100 \%$ of occlusion is obtained. Fig. $10, a_{1}, a_{2}$ show typical occluded images with rates of occlusion of $15 \%$ and $65 \%$ respectively. The image obtained at each step is processed and the retrieved position is compared to the expected one as used for image generation. The reconstructed position is considered valid if the decoded binary sequence fits the correct one.

This protocol is described visually by a short movie clip in supplemental material 4. and was repeated 300 times to gather statistically relevant information. Fig. $10 \mathrm{~b}$ presents the rate of positioning success as a function of the percentage of image occlusion. On the basis of these 300 random trials, a $100 \%$ success rate is observed up to $15 \%$ of occlusion and the probability of correct detection is above $90 \%$ up to $55 \%$ of occlusion. Furthermore, this excellent level of performance against occlusion 


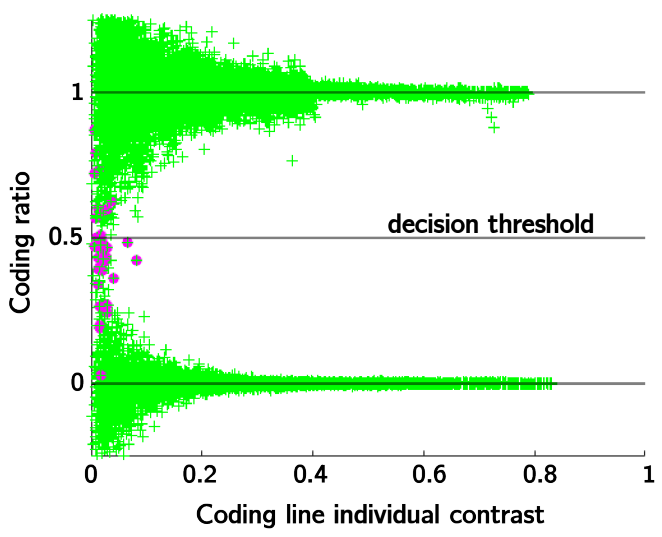

Fig. 9. 2D histogram of thumbnail coding lines contrast and coding ratio. Each coding line of every image from the media 3. is represented according to its contrast and its coding value. As the contrast lowers, coding ratio tends to converge to the decision threshold value. Magenta values represent coding lines that were badly binarized without affecting the decoding process.
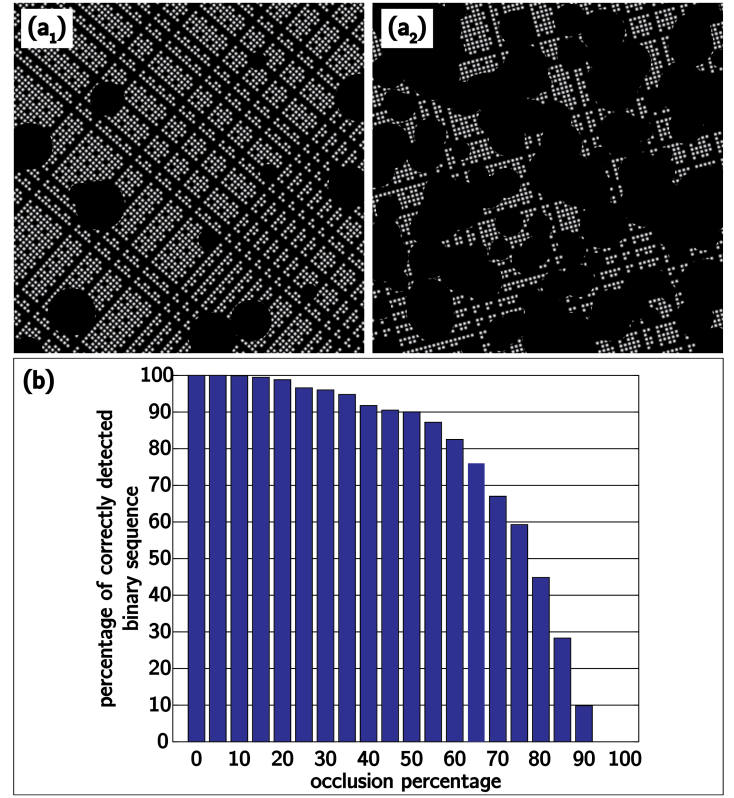

Fig. 10. Decoding robustness to occlusion. $\left(a_{1}\right)$ shows a pattern image with $15 \%$ of occlusion and $\left(a_{2}\right)$ shows a pattern image with $65 \%$ of occlusion. $(b)$ represents the data analysis of a set of 300 pattern images where random occlusions are progressively applied.

can be improved by tuning the bandwidth of the spectral filter to a narrower value. The position reconstruction principle involves indeed a space-frequency trade-off in which the spectral resolution can be increased at the detriment of the eventual positioning resolution and reciprocally. The image distortions resulting from occlusion results in supplementary spectral components that are filtered out more efficiently as the spectral filter becomes narrower. However, for the sake of consistency of the results presented throughout the paper, simulations with occlusion were carried out with the nominal width of the spectral filter; i.e. the value that allows a positioning resolution below one nanometer as presented previously.

\section{CONClusion}

This paper investigates the robustness of a computer vision method for the measurement of the 3-DOF in-plane position of a target carrying a pseudo-periodic pattern. The periodic property of the pattern allows a high degree of interpolation with respect to both the physical pattern period and the image pixel frame. This processing is based on phase computations and results in a high resolution, typically one nanometer in lateral displacement and one microradian in rotation with a $10 \times$ magnification objective and from a pattern period of $9 \mu \mathrm{m}$. A specificity of the method is to use the linear phase data for the Boolean decoding of the absolute order of the pattern lines and columns that are encrypted within a binary linear feedback shift register sequence. That phase data provides an a priory knowledge of the location of the pattern features of interest and this allows to determine the binary encoded position with a locally optimized decision threshold. This phase-based decoding is 
explained in full details in section $[\mathrm{II}]$ and confers an excellent robustness to the method as demonstrated throughout the paper by both experiments and simulations. These experiments were developed to fit as much as possible reality of computer-vision robustness and the described method was proved to be robust to different kinds of image damaging effects such as poor lighting conditions, noising, blur and occlusions. The demonstrated robustness complements the high resolution and extra-large measurement range of the proposed method that constitutes a precious tool for visual 3-DOF position control available for diverse research or industrial applications. This measurement method has already been used in our laboratory to control the planar displacements of a serial robot in the frame of an automatized assembly task. The method is readily applicable to the characterization of $X, Y, \Theta$ manipulators, either at a calibration stage or when used within visual servoing loops [14]. This characterization function is important, especially at the micro-scale where stage sensors are not representative of the actual position of the end-effector of the robot. Moreover, the scalable property of the method allows the tuning of the resolution and the range at the desired levels. Our demonstration is based on a 10x microscope objective leading to a $1 \mathrm{~nm}$ resolution over $10 \mathrm{~cm}$. The choice of a larger elementary period would shift the resolution in the micrometer or millimeter ranges with the same robustness capabilities. In such cases, the lower magnification needed would result in extended field of observation, depth of focus and standoff from the scene. We are currently investigating the application of the method to control the planar position of coordinate measuring machines. As the presented method is proved to be robust to acquisition alteration and allows a $10^{8}$ range-to-resolution ratio for in plane measurement, further studies will focus on the extension of the measure along 6-DOF with the same pattern encoding method. One may also notice that the thumbnail images provide information of the local signal-to-noise ratio. This knowledge could be used to select the most-informative pixels to be considered in the computation of the unwrapped phase plane equations with further improvement of the method robustness, especially in the case of very low quality images. This possibility will be explored in further applications. At the current stage, the accuracy of the method relies on the sole fabrication of the pattern and the measure is therefore self calibrated. Fabrication uncertainty can lead to accuracy issues and is currently under investigation with a laboratory of metrology to qualify the uncertainty associated.

\section{ACKNOWLEDGMENT}

This work was supported by ROBOTEX (ANR-10-EQPX-44-01), by Cross-disciplinary Research (EIPHI) Graduate School (contract ANR-17-EURE-0002), the French Investissements d'Avenir program and the I-SITE Bourgogne Franche-Comté (BFC) project (contract ANR-15-IDEX-03). The encoded target was realized thanks to the RENATECH technological network and its FEMTO-ST facility MIMENTO. Authors acknowledge G. Jutzi, L. Robert, M. Suarez and L. Gauthier-Manuel for technological and experimental assistance.

\section{REFERENCES}

[1] X. Xu, M. Zhao, and J. Lin, "Detecting weak position fluctuations from encoder signal using singular spectrum analysis," ISA transactions, vol. 71, pp. $440-447,2017$.

[2] J. Lin and C. Chen, "Positioning and tracking of a linear motion stage with friction compensation by fuzzy logic approach," ISA transactions, vol. 46, no. 3, pp. 327-342, 2007.

[3] A. Safa, R. Y. Abdolmalaki, S. Shafiee, and B. Sadeghi, "Adaptive nonsingular terminal sliding mode controller for micro/nanopositioning systems driven by linear piezoelectric ceramic motors," ISA transactions, vol. 77, pp. 122-132, 2018.

[4] Z. Chen, H. Pu, X. Liu, D. Peng, and Z. Yu, "A time-grating sensor for displacement measurement with long range and nanometer accuracy," IEEE Transactions on Instrumentation and Measurement, vol. 64, no. 11, pp. 3105-3115, 2015.

[5] Z. Yu, K. Peng, X. Liu, H. Pu, and Z. Chen, "A new capacitive long-range displacement nanometer sensor with differential sensing structure based on time-grating," Measurement Science and Technology, vol. 29, no. 5, p. 054009, 2018.

[6] W. Gao, S.-W. Kim, H. Bosse, H. Haitjema, Y. Chen, X. Lu, W. Knapp, A. Weckenmann, W. Estler, and H. Kunzmann, "Measurement technologies for precision positioning," CIRP Annals, vol. 64, no. 2, pp. 773-796, 2015.

[7] M. Torralba, M. Valenzuela, J. Yagüe-Fabra, J. Albajez, and J. Aguilar, "Large range nanopositioning stage design: A three-layer and two-stage platform," Measurement, vol. 89, pp. 55-71, 2016.

[8] X. Li, W. Gao, H. Muto, Y. Shimizu, S. Ito, and S. Dian, "A six-degree-of-freedom surface encoder for precision positioning of a planar motion stage," Precision Engineering, vol. 37, no. 3, pp. 771-781, 2013.

[9] H.-L. Hsieh and S.-W. Pan, "Development of a grating-based interferometer for six-degree-of-freedom displacement and angle measurements," Optics express, vol. 23, no. 3, pp. 2451-2465, 2015.

[10] M. Carreras, P. Ridao, R. García, and T. Nicosevici, "Vision-based localization of an underwater robot in a structured environment," in 2003 IEEE International Conference on Robotics and Automation (Cat. No. 03CH37422), vol. 1. IEEE, 2003, pp. 971-976.

[11] S. Saito, A. Hiyama, T. Tanikawa, and M. Hirose, "Indoor marker-based localization using coded seamless pattern for interior decoration," in 2007 IEEE Virtual Reality Conference. IEEE, 2007, pp. 67-74.

[12] C.-C. Hsu, M.-C. Lu, W.-Y. Wang, and Y.-Y. Lu, "Distance measurement based on pixel variation of ccd images," ISA transactions, vol. 48, no. 4, pp. 389-395, 2009

[13] C. Liu, Y. Yuan, and M. Zhang, "Uncertainty analysis of displacement measurement with imetrum video gauge," ISA transactions, vol. 65, pp. 547-555, 2016.

[14] B. Mauzé, R. Dahmouche, G. J. Laurent, A. N. André, P. Rougeot, P. Sandoz, and C. Clévy, "Nanometer precision with a planar parallel continuum robot," IEEE Robotics and Automation Letters, vol. 5, no. 3, pp. 3806-3813, 2020.

[15] C. Yamahata, E. Sarajlic, G. J. Krijnen, and M. A. Gijs, "Subnanometer translation of microelectromechanical systems measured by discrete fourier analysis of ccd images," Journal of Microelectromechanical Systems, vol. 19, no. 5, pp. 1273-1275, 2010.

[16] C. Zhao, C. F. Cheung, and M. Liu, "Nanoscale measurement with pattern recognition of an ultra-precision diamond machined polar microstructure," Precision Engineering, vol. 56, pp. 156-163, 2019.

[17] A. N. Andre, P. Sandoz, B. Mauze, M. Jacquot, and G. J. Laurent, "Sensing one nanometer over ten centimeters: A micro-encoded target for visual in-plane position measurement," IEEE/ASME Transactions on Mechatronics, vol. 25, no. 3, pp. 1193-1201, 2020. 
[18] W. Shang, H. Lu, W. Wan, T. Fukuda, and Y. Shen, "Vision-based nano robotic system for high-throughput non-embedded cell cutting," Scientific reports, vol. 6 , p. 22534, 2016.

[19] J. Liu, Z. Gong, K. Tang, Z. Lu, and Y. Sun, "Locating end-effector tips in automated micromanipulation," in 2013 IEEE International Conference on Robotics and Automation. IEEE, 2013, pp. 1724-1729.

[20] H. K. Chu, J. K. Mills, and W. L. Cleghorn, "Dual-arm micromanipulation and handling of objects through visual images," in 2012 IEEE International Conference on Mechatronics and Automation. IEEE, 2012, pp. 813-818.

[21] E. Avci, C.-N. Nguyen, K. Ohara, M. Kojima, Y. Mae, and T. Arai, "Towards high-speed automated micromanipulation," in 2013 IEEE International Conference on Robotics and Automation. IEEE, 2013, pp. 1718-1723.

[22] H. Yang, S. Zheng, J. Lu, and Z. Yin, "Polygon-invariant generalized hough transform for high-speed vision-based positioning," IEEE Transactions on Automation Science and Engineering, vol. 13, no. 3, pp. 1367-1384, 2016.

[23] J. Kokorian, F. Buja, and W. M. van Spengen, "In-plane displacement detection with picometer accuracy on a conventional microscope," Journal of Microelectromechanical Systems, vol. 24, no. 3, pp. 618-625, 2014.

[24] L. Hostettler, A. Özgür, S. Lemaignan, P. Dillenbourg, and F. Mondada, "Real-time high-accuracy 2d localization with structured patterns," in 2016 IEEE International Conference on Robotics and Automation (ICRA). IEEE, 2016, pp. 4536-4543.

[25] G. Bomarito, J. Hochhalter, T. Ruggles, and A. Cannon, "Increasing accuracy and precision of digital image correlation through pattern optimization," Optics and Lasers in Engineering, vol. 91, pp. 73-85, 2017.

[26] H. Li, B. Zhu, Z. Chen, and X. Zhang, "Realtime in-plane displacements tracking of the precision positioning stage based on computer micro-vision," Mechanical Systems and Signal Processing, vol. 124, pp. 111-123, 2019.

[27] N. Yoshimizu, A. Lal, and C. R. Pollock, "Nanometrology optical ruler imaging system using diffraction from a quasiperiodic structure," Optics express, vol. 18 , no. 20, pp. 20827-20838, 2010.

[28] Z.-H. Chen and P. S. Huang, "A vision-based method for planar position measurement," Measurement Science and Technology, vol. 27, no. 12, p. 125018, 2016.

[29] H. Sugiura, S. Sakuma, M. Kaneko, and F. Arai, "On-chip method to measure mechanical characteristics of a single cell by using moiré fringe," Micromachines, vol. 6, no. 6, pp. 660-673, 2015.

[30] V. Guelpa, G. Laurent, P. Sandoz, J. Zea, and C. Clévy, "Subpixelic measurement of large 1d displacements: Principle, processing algorithms, performances and software," Sensors, vol. 14, no. 3, pp. 5056-5073, 2014.

[31] P. Masa, E. Franzi, and C. Urban, "Nanometric resolution absolute position encoders," CSEM Scientific and Technical Report, pp. 1-3, 2008.

[32] J.-A. Kim, J. W. Kim, C.-S. Kang, and J. Jin, "Note: An absolute xy- $\theta$ position sensor using a two-dimensional phase-encoded binary scale," Review of Scientific Instruments, vol. 89, no. 4, p. 046105, 2018.

[33] J.-A. Galeano-Zea, P. Sandoz, E. Gaiffe, J.-L. Prétet, and C. Mougin, "Pseudo-periodic encryption of extended 2-d surfaces for high accurate recovery of any random zone by vision," International Journal of Optomechatronics, vol. 4, no. 1, pp. 65-82, 2010.

[34] J. Stevenson and J. Jordan, "Absolute position measurement using optical detection of coded patterns," Journal of Physics E: Scientific Instruments, vol. 21 , no. 12 , p. 1140,1988 .

[35] J.-A. Kim, J. W. Kim, C.-S. Kang, J. Jin, and T. B. Eom, "Note: Nonlinearity error compensated absolute planar position measurement using a twodimensional phase-encoded binary grating," Review of Scientific Instruments, vol. 84, no. 5, p. 056102, 2013.

[36] S. W. Golomb et al., Shift register sequences. Aegean Park Press, 1967.

[37] J.-Y. Rauch, O. Lehmann, P. Rougeot, J. Abadie, J. Agnus, and M. A. Suarez, "Smallest microhouse in the world, assembled on the facet of an optical fiber by origami and welded in the $\mu$ robotex nanofactory," Journal of Vacuum Science \& Technology A: Vacuum, Surfaces, and Films, vol. 36, no. 4, p. 041601, 2018

[38] E. Krotkov, "Focusing," International Journal of Computer Vision, vol. 1, no. 3, pp. 223-237, 1988.

[39] S. Pertuz, D. Puig, and M. A. Garcia, "Analysis of focus measure operators for shape-from-focus," Pattern Recognition, vol. 46, no. 5, pp. 1415-1432, 2013. 